\title{
New Control Strategy for Energy Conversion Based on Coupled Magnetic Structures
}

\author{
M.C.González*, L.Laguna*, P.Alou*, O.García*, J.A.Cobos* and H.Visairo** \\ *Universidad Politécnica de Madrid/Centro de Electrónica Industrial, Madrid, España \\ ** Intel Corporation/Systems Research Center, Systems Technology Lab, Guadalajara, México
}

\begin{abstract}
In this paper, a new strategy for energy conversion based on a coupled magnetic structure is presented. A proper control of the input voltages provides constant output voltage at any time and ideally no output filter is required and no energy is stored, enabling very fast dynamics and low losses in the converter since switching frequency can be very small. Ideal features and actual limitations of the proposed concept are analyzed. A prototype with a two-input magnetic structure is built in order to prove the concept.
\end{abstract}

\section{INTRODUCTION}

This paper proposes a new concept to transfer energy. It is based on coupled magnetic structures like those used in multiphase converters with coupled inductors and the associated magnetic structures [2-3,6-9]. These solutions can require a complex magnetic structure, but they provide interesting advantages regarding dynamic response and efficiency. These solutions can be represented (Figure 1) by a multi-input magnetic structure where the instantaneous output voltage is the average value of all the instantaneous input voltages.

These converters with coupled magnetic structures take advantage of the current ripple reduction obtained when coupling the inductors in a multiphase Buck converter. As stated in [[1], [2]] the current ripple is reduced without affecting the dynamic response of the converter. Also, it is necessary to have enough inductance in order to filter the output voltage. The filter inductance (Figure 2) is usually provided by the leakage inductance $\left(L_{L K}\right)$ of the transformer [1]. This inductance stores energy and provides regulation capability, but also limits the dynamic response. The necessary amount of stored energy (and therefore the needed value of $L_{L K}$ ) would depend on the design of the converter (switching frequency, current ripple, magnetizing inductance, etc). The higher the value of $L_{L K}$, the higher the amount of stored energy and the slower the dynamic response of the converter.

In this paper, a new control strategy that will allow a coupled multiphase Buck converter to operate (ideally) without output filter and therefore without energy storage is presented. Proposed control strategy: If the sum of input voltages is

This work was supported by Intel Corporation.

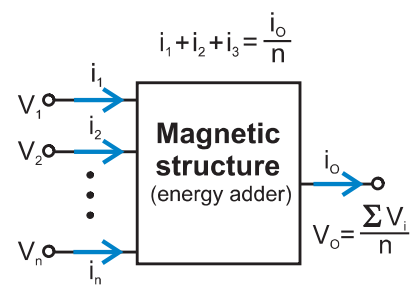

Fig. 1. Concept ilustration: $n$ inputs magnetic structure

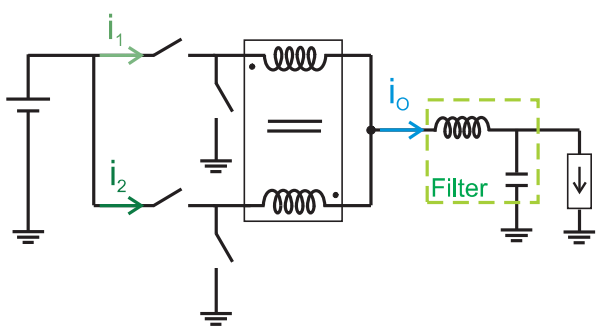

Fig. 2. State of the art multiphase Buck converter with coupled inductors store energy in the filter inductance

constant at any time, then the output voltage is also constant at any time and ideally no output filter is required. This constant input - constant output control strategy, provides interesting advantages from the point of view of dynamic response and efficiency. This conversion strategy presents important differences with conventional DC-DC converters. Since it is not necessary to store energy with the proposed control strategy, $L_{L K}$ should be as low as possible. This would result in very fast dynamic response.

Ideal operation principle and features of the proposed topology are presented in Section II. Section III highlights the practical issues that arise when the concept is actually implemented. Details of the design and preliminary design criteria are shown in Section IV and the experimental results of the concept validation are presented in Section V.

\section{Proposed Concept: IdeAl Operation AND FEATURES}

Before introducing the general concept, a two-input design is presented. For this case, the control strategy to achieve constant output voltage at any time is: in the first half of a 


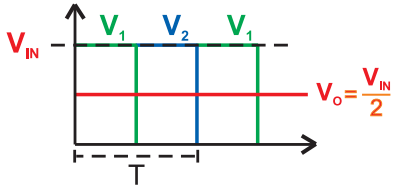

a)

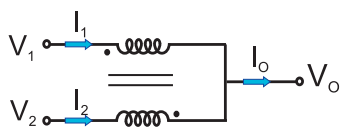

b)
Fig. 3. a)Instantaneous value of input and output voltages, b)Two-input magnetic structure

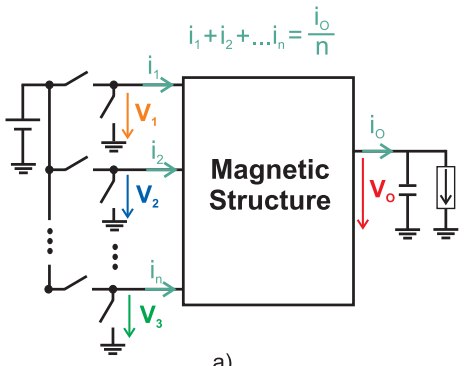

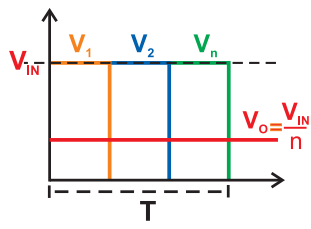

b)
Fig. 4. Case for $\mathrm{n}$ inputs converter

time interval $\mathrm{T}$, the value of the voltage of input 1 is equal to a constant DC voltage $\left(V_{I N}\right)$, while the voltage value in the input 2 is zero, and in the second half of $\mathrm{T}$, the voltage of input 1 is equal to zero while the voltage value in the input 2 is equal to $V_{I N}$. This is illustrated in Figure 3a). Analyzing the magnetic structure in Figure $3 b$ ), it can be seen that the voltage in the magnetic structure, at any time, is

$$
v_{1}-v_{O}=v_{O}-v_{2}
$$

therefore output voltage is given by

$$
v_{o}=\frac{v_{1}+v_{2}}{2}
$$

at any instant of time and the average voltage value for this particular case is $\frac{V_{I N}}{2}$. In the same way, both input currents are equal at any instant of time, since the output current is

$$
i_{O}=i_{1}+i_{2}
$$

and $i_{1}=i_{2}$ for this particular case it is given that $i_{1}=i_{2}=\frac{i_{O}}{2}$ for any instant of time.

\section{A. General operation principle}

The sum of the voltage inputs applied to the magnetic structure, like the one shown in Figure 4 must be constant at any instant of time:

$$
\sum v_{i}=\text { constant } \forall t
$$

then the output voltage keeps constant at any instant of time $\left(v_{o}=\frac{\sum v_{i}}{n}\right)$ and ideally no output filter is required. This condition can be achieved by shifting the input voltage waveforms applied to the magnetic structure and being the duty cycle a multiple of the inverse of the number of inputs $\left(d=k \cdot \frac{1}{n}\right)$.

\section{B. Operation Features}

The main features of this conversion strategy are:

1) Dynamic response and efficiency. Since "no energy" is stored, very fast dynamic response can be accomplished operating at low switching frequency, which can be selected to optimize efficiency without affecting the dynamic response. A simulation for this ideal case is presented in Figure 5. The voltage drop after the load step is due to the equivalent series resistance.

2) Efficiency. The efficiency of this topology can be very high since the converter may operate at very low switching frequency without limiting the dynamic response. The main losses of this converter are due to conduction losses since switching losses can be really small thanks to the very low switching frequency (for example $40 \mathrm{kHz}$ ). Therefore, the efficiency depends mainly on the DC output impedance (equivalent series resistance).

3) Regulation capability. This solution has limited regulation capability since "no energy" is stored. The proposed converter operates on the nodes, being the nodes the points of operation where the duty cycle is a multiple of $\frac{1}{n}$ at any time:

$$
d=k \cdot \frac{1}{n}
$$

where $d$ is the duty cycle, $n$ the number of inputs to the magnetic structure and $k$ is an integer number that ranges from " 0 " up to the number of inputs " $n$ " $(k=0,1,2 \ldots, n)$.

Duty cycle can not be slightly modified to regulate deviations of the output voltage. Therefore, the output voltage can only be adjusted within discrete steps:

$$
v_{o}=d \cdot V_{I N}=k \cdot \frac{V_{I N}}{n}
$$

Depending on the "input voltage to output voltage" ratio, the control must adjust the duty cycle to be a multiple of $\frac{1}{n}$. This functionality of the control can be easily implemented with Digital Control. The regulation of this converter works like a discrete regulation. The higher the number of inputs, the better the resolution of the discrete regulation. The discrete regulation concept can be seen as a duty-cycle-selectable Power DAC (Power Digital-Analog Converter). Several applications are possible, ranging from wide input voltage converters (i.e. Vin=1-12V) to Dynamic Voltage Scaling (DVS). 

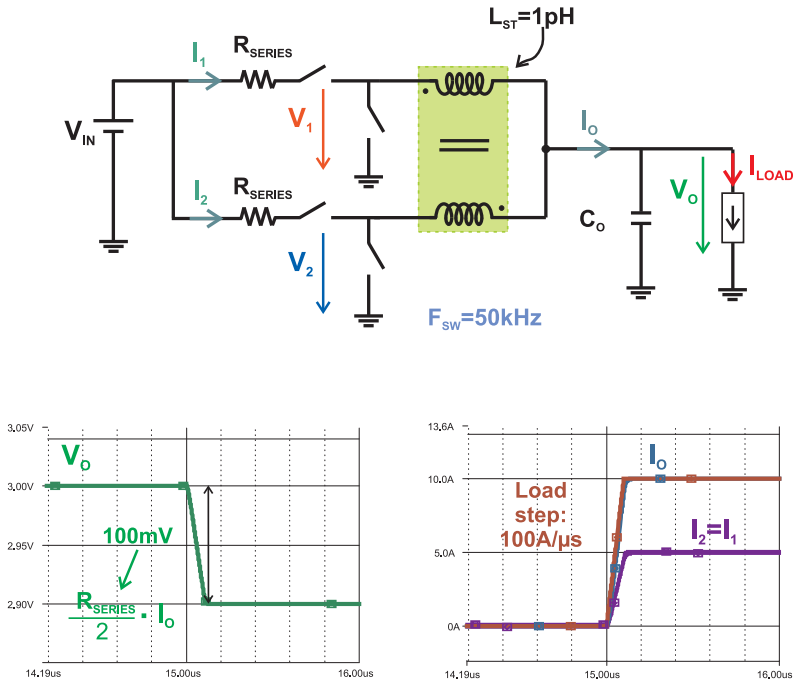

Fig. 5. Simulation with "ideal" parameters, and dynamic response of this ideal circuit

The load regulation depends on the open loop output impedance of the converter. Depending on the intended system architecture, fine load regulation can be achieved by using a pre-regulator or a post-regulator combined with this "DC/DC transformer".

\section{ACtual Limitations And FeAtures}

Three main limitations arise when this concept is actually built:

1) In fact, the concept of "no energy storage" can not be achieved, since a leakage inductance is always present in the construction of the transformer. A very small leakage inductance can be reached with a very good coupled magnetic structure and the dynamic response can still be very fast although it is not instantaneous. The simulation of a real converter with a feasible leakage inductance is shown in Figure 6. Output voltage deviation is $210 \mathrm{mV}$ when the load step occurs $(100 \mathrm{~A} / \mu \mathrm{s})$, this is obtained with only $22 \mu \mathrm{F}$ output capacitance ( $1 \mathrm{x} 22 \mu \mathrm{F}-500 \mathrm{pH}-3 \mathrm{~m} \Omega)$. The simulated converter still responds with a high current slew rate $(10 \mathrm{~A} / \mu \mathrm{s})$ being the leakage inductance $L_{S T}=40 \mathrm{nH}$ ( $L_{S T}$ is the short-circuited test inductance, measured from one winding when the other is short-circuited). To achieve a similar response $(10 \mathrm{~A} / \mu \mathrm{s})$ with an equivalent Buck converter, it would be required a bandwidth around $250 \mathrm{kHz}$. This calculation is done based on the equation represented in [3] $\left(\Delta B(H z)=\frac{1}{4 \cdot t_{r}}\right)$ that relates the system bandwidth to the slew rate of the inductor current. Therefore, the response obtained with a two-input magnetic structure working at $50 \mathrm{kHz}$ would be achievable with an equivalent buck working at approximately $800 \mathrm{kHz}$ of switching frequency $(250 \mathrm{kHz}$

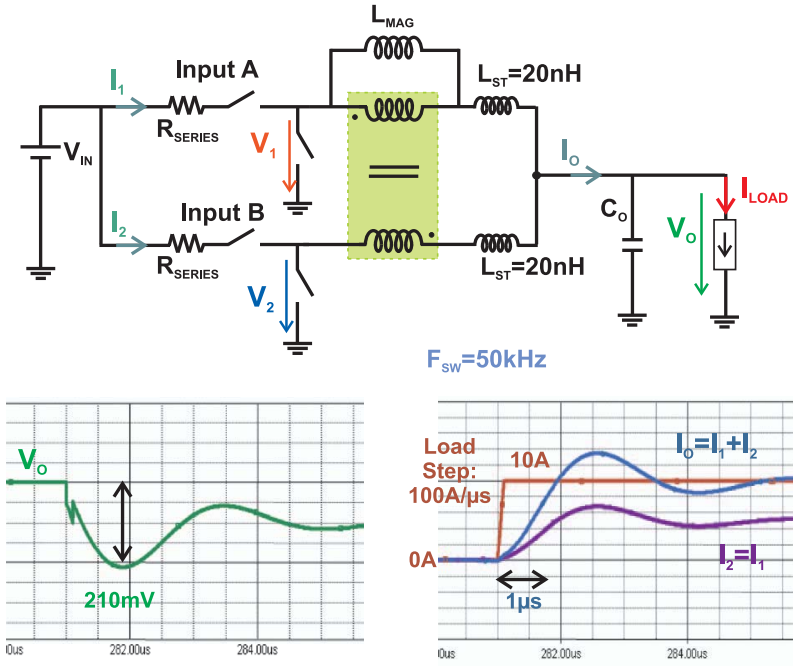

Fig. 6. Two-input magnetic structure simulation, with a feasible leakage inductance $\left(L_{S T}\right)$

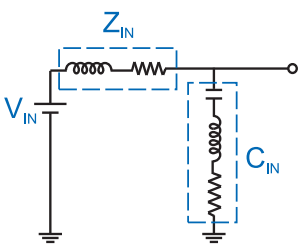

Fig. 7. Model of the input impedance of the converter

bandwidth).

2) In the simulation shown in Figure 6, it is assumed an ideal voltage source at the input of the converter. However, in the real prototype an impedance would be associated to the input source of the converter and connection cables $\left(Z_{I N}\right.$ in Figure 7). Since the amount of energy stored in the magnetic structure and output capacitor (output filter of the converter) is very small, this impedance at the input of the converter becomes important and slows down the energy transfer between input and output of the converter. The influence of this input impedance is compensated by storing energy in an input capacitor $\left(C_{I N}\right.$ in Figure 7). Two different values of input capacitors $\left(C_{I N}\right)$ that would compensate the influence of $Z_{I N}$ are presented in Table I. Both cases presented in this table would result in very similar dynamic responses. The dynamic response of the converter with an input impedance of $Z_{I N}=80 m \Omega-2.5 \mu H, C_{I N}=2.2 m F-17 n H-6 m \Omega$ is shown in the simulation of Figure 8.

3) In section II, it is said that the instantaneous output voltage is equal to the sum of the instantaneous voltages in the inputs (v1 and v2). However, commutations are not ideal, then the sum of the instantaneous input 
TABLE I

CORRESPONDING VALUES OF $C_{I N}$ FOR TWO CASES OF INPUT IMPEDANCES $\left(Z_{I N}\right)$

\begin{tabular}{|c||c|}
\hline$Z_{I N}$ & $C_{I N}$ \\
\hline $80 m \Omega-2.5 \mu H$ & $2.2 m F-17 n H-6 m \Omega$ \\
\hline $80 m \Omega-250 n H$ & $220 \mu F-6 n H-55 m \Omega$ \\
\hline
\end{tabular}
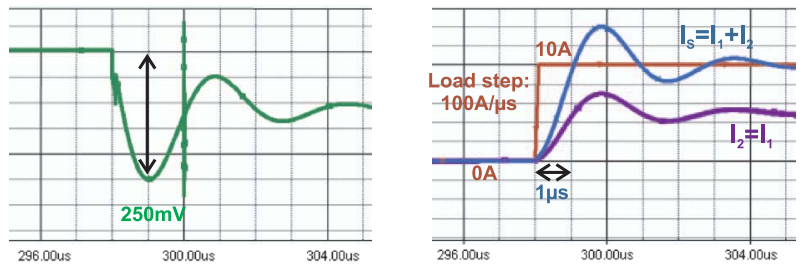

Fig. 8. Response of the converter to a $10 \mathrm{~A}$ load step $(100 \mathrm{~A} / \mu \mathrm{s})$ considering the influence of a non-ideal input voltage source

voltage is not perfectly constant and voltage oscillations can appear at the output. Figure 9b) and 9c) show two commutations that produces excess or lack of voltage at the input, respectively. This non-ideal commutation generates voltage ripple at the output. This ripple will be called switching ripple (Figure 16). The worse the commutation is, the bigger the ripple that appears in the output. Therefore, a priority when choosing a control implementation should be the practical achievement of fast rise and fall times of the driving signal for each (high side mosfet) input in order to minimize the switching ripple.

\section{DESIGN CRITERIA}

\section{A. Switching Frequency.}

The selected operating frequency range of the proposed converter is in the tenths of $\mathrm{kHz}$. In order to analytically determine the frequency for which the total losses are minimized, a very simple losses model is used. The switching losses are defined as follows:

$$
P_{S W}=P_{C O S S}+P_{T U R N \_O N}+P_{T U R N \_O F F}+P_{D R R}
$$

Where $P_{C O S S}$ is:

$$
P_{C O S S}=\frac{1}{2} \cdot C_{O S S} \cdot V_{I N}^{2} \cdot f_{S W}
$$

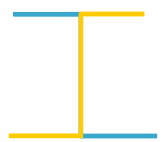

a)

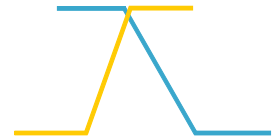

b)

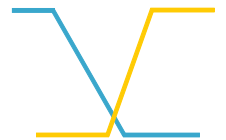

Fig. 9. a) Ideal commutation. b) Commutation resulting with excess of voltage at the output. c) Commutation resulting with lack of voltage at the output.

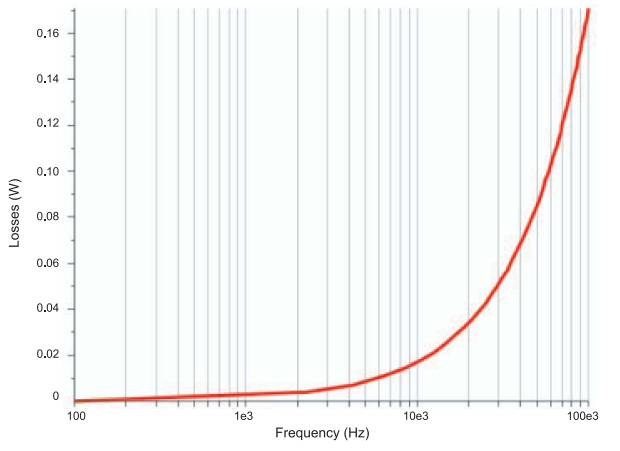

Fig. 10. Analytical model prediction for switching losses

$P_{T U R N \_O N}, P_{T U R N \_O F F}$, are defined as:

$$
\begin{aligned}
P_{T U R N \_O N} & =\int_{0}^{\text {trise }} v_{O N} \cdot i_{O N} d t \\
P_{T U R N \_O F F} & =\int_{0}^{t f a l l} v_{O F F} \cdot i_{O F F} d t
\end{aligned}
$$

and $P_{D R R}$ is the body diode reverse recovery loss ([4]):

$$
P_{D R R}=V_{I N} \cdot Q_{R R} \cdot f_{S W}
$$

The proposed converter operates at very low switching frequency, so the most important losses are the conduction and circulating energy losses which are given by:

$$
P_{C O N D}=P_{R D S o n}+P_{T_{-} C O R E}+P_{T_{-} W I N D I N G}
$$

and total losses would be defined as:

$$
P_{\text {TOTAL }}=P_{S W}+P_{C O N D}
$$

According to this simple model, switching losses are increased when the switching frequency is increased, as shown in the graph in Figure 10. Conduction losses are mainly dependent on the average and ripple values of the current. Therefore, increasing the switching frequency decreases the current ripple value and conduction losses are reduced (Figure 11). According to the chosen inductance, and MOSFETs, there is a frequency for which both switching and conduction losses are minimum. A comparison between the model prediction and the measurements of the prototype for full load (10A) is shown in Figure 12. Although the optimum frequency predicted by the model $(10 \mathrm{kHz})$ does not match the actual point of minimum losses, which is around $40 \mathrm{kHz}$ (as shown in the next section), the optimum frequency is in the tenths of $\mathrm{kHz}$ in both cases. 


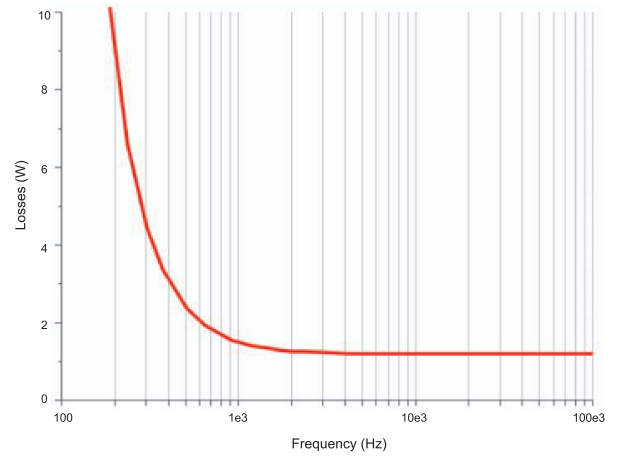

Fig. 11. Conduction losses predicted by analytical model

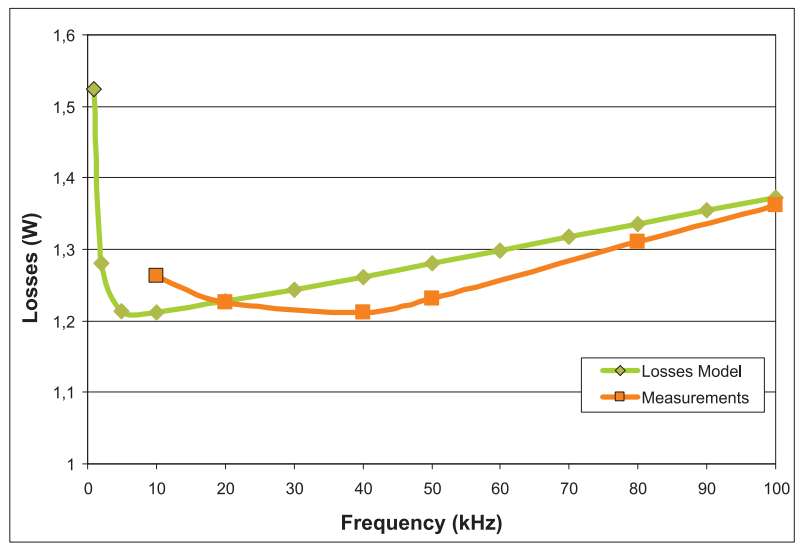

Fig. 12. Comparation between calculated and measured losses at full load (30W)

\section{B. Magnetic Structure.}

The design of the magnetic structure used in the proposed converter takes into account three main requirements.

1) Trade-off between magnetizing inductance and losses. Magnetizing inductance needs to be high enough to avoid excessive losses and saturation of the magnetic core when working at low frequency (tenths of $\mathrm{kHz}$ ). However it is always preferable to use a small core. So a trade-off between circulating energy losses and transformer core size has to be taken into consideration. For this prototype, an RM6-3F3 core was chosen.

2) Minimum energy storage. The use of interleaving techniques between windings results in lower leakage inductance value. In this case, four paralleled primaries and four paralleled secondaries have been interleaved. No

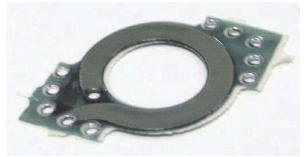

Fig. 13. The magnetic structure was built with stacked PCBs connected in series

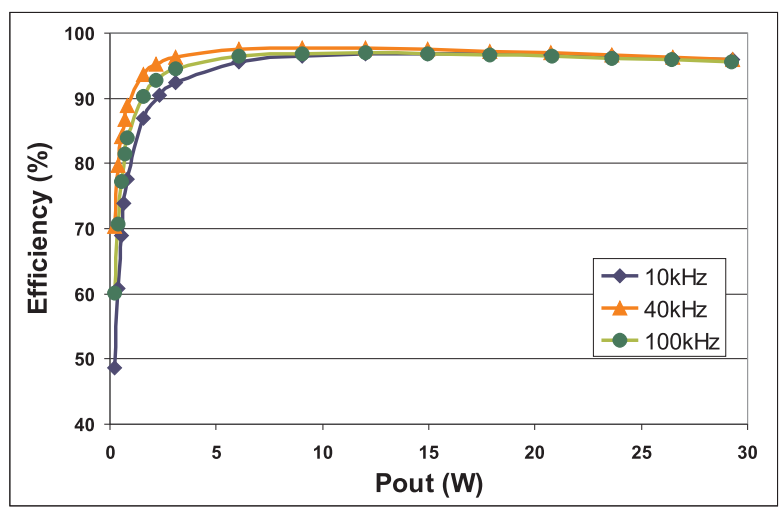

Fig. 14. Efficiency measured in the prototype for different frequencies for full load range

gap is used.

3) Low equivalent series resistance. Since the main losses in the proposed converter, are conduction losses, the contribution of the transformer windings to the total series resistance needs to be minimum. This can be done by paralleling as many windings as possible in the window area of the RM core.

The validation of the design of the magnetic structure is done with PEmag [4] and a design methology for coupled inductor multiphase converters can be found in [5]. The magnetic structure used in this prototype was build using stacked PCB windings (Figure 13).

\section{MOSFETs considerations.}

As said above, the main consideration when choosing MOSFETs should be a low $R_{D S o n}$. Since switching frequency is very small, a MOSFET with very low RDSon can be used despite the high $C_{I S S}$ usually associated. For this prototype IRF7457 MOSFETs have been used.

\section{EXPERIMENTAL VALIDATION}

In order to validate the concept, a two-input magnetic structure was built. This converter operates with an input voltage of $6 \mathrm{~V}$. Since it has a magnetic structure with only two inputs, it operates at a node with $50 \%$ of duty cycle; therefore the output voltage is $3 \mathrm{~V}$. Maximum output current is $10 \mathrm{~A}$. As said above, it operates at very low switching frequency $(40 \mathrm{kHz})$, and the magnetic structure used is a highly coupled transformer being $40 \mathrm{nH}$ the inductance measured in the short circuited test.

This prototype presents very high efficiency, over $90 \%$ among a wide load range, from $1 \mathrm{~W}$ to $30 \mathrm{~W}$, and with a peak efficiency of $97.7 \%$. Efficiency above $95 \%$ was achieved from $2 \mathrm{~W}$ to $30 \mathrm{~W}$. These efficiency measurements are shown in the graphs of Figure 14. A detail for light load measurements 


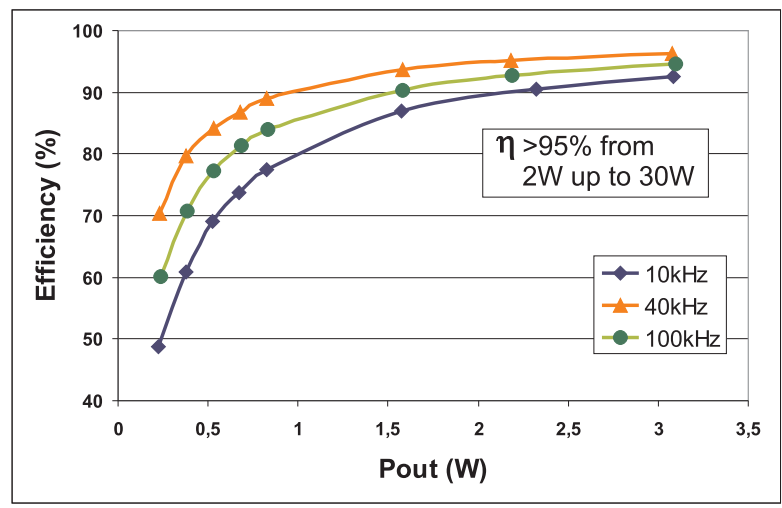

Fig. 15. Light Load efficiency measured from $230 \mathrm{~mW}$ to $2 \mathrm{~W}$

is shown in Figure 15. From this graph it can be seen that the selection of the switching frequency is very important. Taking as reference $230 \mathrm{~mW}$ of output power it can be seen that the efficiency for a switching frequency of $40 \mathrm{kHz}$ is higher than that for $10 \mathrm{kHz}$, this is because in this point the major losses are due to circulating energy in the magnetizing inductance. At $100 \mathrm{kHz}$, however the switching losses become more important and the efficiency is worse.

As explained in section III, the duty cycle needs to be very precise and the commutations must be, as near as possible, ideal commutations. Digital control was used to test the prototype in open loop. The measured waveforms in Figure 16 show the commutation ripple in the output voltage and the driving signal for the high side transistor of each input. Figure 17 shows the converter response under a $10 \mathrm{~A}$ load step $(40 \mathrm{~A} / \mu \mathrm{s})$. Voltage deviation is $330 \mathrm{mV}$, being the output capacitance only $22 \mu \mathrm{F}$. Thanks to the low leakage inductance $(40 \mathrm{nH})$ the converter response is very fast since the return to the nominal voltage value takes only around $2 \mu \mathrm{s}$ after the load step. Since the leakage inductance is very small, the energy stored at the input also affects the output deviation. The design of the series inductance becomes a trade off between the dynamic response and the switching ripple. The lower the series inductance, the better the dynamic response but also the higher the switching ripple. The proper driving of the high side switches becomes very important to reduce this switching ripple.

Regarding the actual limitations of the presented concept, two options seem feasible to apply this concept: Option 1, "DC-DC transformer" + post-regulator, would be a high efficiency solution, providing a low voltage at the input of the post regulator that would increase the efficiency of this second converter. Option 2, Pre-regulator + "DC-DC transformer", this solution would take advantage of the fast response of the proposed concept. The appropriate operation of this second option strongly depends on minimizing the switching ripple

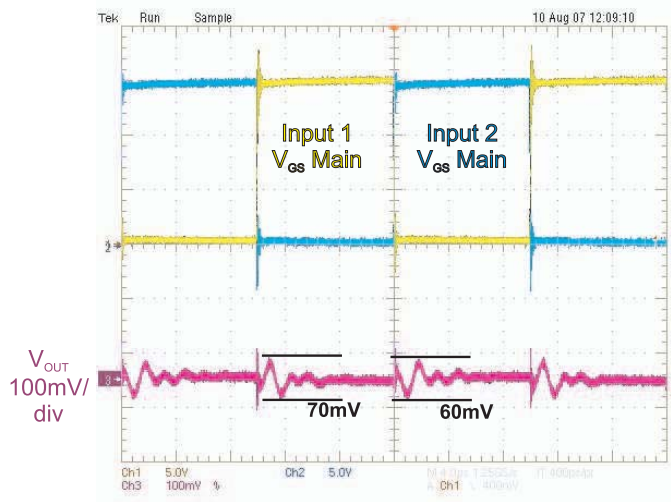

Fig. 16. Measured output voltage ripple: driving signals 5V/div, output voltage $50 \mathrm{mV} / \mathrm{div}$ and $4 \mu \mathrm{s} / \mathrm{div}$.

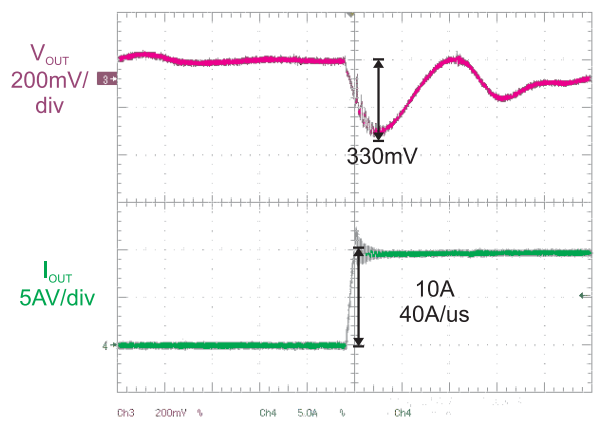

Fig. 17. Output voltage deviation $(200 \mathrm{mV} / \mathrm{div})$ when load steps from $0 \mathrm{~A}$ up to $10 \mathrm{~A}$ step $(5 \mathrm{~A} / \mathrm{div})$.

and reducing the voltage deviation during load steps by increasing the number of output capacitors.

\section{CONCLUSION}

The proposed strategy conversion acts like a "DC/DC transformer" where the output voltage can be controlled within discrete steps. The switching frequency can be selected to optimize efficiency without affecting the dynamic response; switching frequency and dynamic response are uncoupled. Since, ideally no energy is stored, dynamic response can be very fast, being limited by the leakage inductance. The load regulation is limited by the open loop output impedance of the converter. The concept was validated on a $6 \mathrm{~V}$ to $3 \mathrm{~V}$, $10 \mathrm{~A}$ two-input magnetic structure operating at $40 \mathrm{kHz}$. A very high efficiency is obtained over a wide load range $(>95 \%$ from $2 W$ up to $30 W$ ). Despite of the leakage inductance $\left(L_{S T}=40 \mathrm{nH}\right)$, the converter responses fast: under a $10 \mathrm{~A}$ load step, the output voltage recovers its nominal value within $2 \mu \mathrm{s}$ and the voltage deviation is $330 \mathrm{mV}$ with only $22 \mu \mathrm{F}$ of output capacitance. A very good synchronization of high side switches is needed to minimize the voltage ripple at the output. Digital control is recommended to manage appropriately the voltages applied to the magnetic structure. 


\section{REFERENCES}

[1] J. Li, C. Sullivan, and A. Schultz, "Coupled-inductor design optimization for fast-response low-voltage dc-dc converters," Applied Power Electronics Conference and Exposition, 2002. APEC 2002. Seventeenth Annual IEEE, vol. 2, pp. 817-823 vol.2, 2002.

[2] P.-L. Wong, P. Xu, P. Yang, and F. Lee, "Performance improvements of interleaving vrms with coupling inductors," Power Electronics, IEEE Transactions on, vol. 16, no. 4, pp. 499-507, Jul 2001.

[3] J. Li, A. Stratakos, A. Schultz, and C. Sullivan, "Using coupled inductors to enhance transient performance of multi-phase buck converters," Applied Power Electronics Conference and Exposition, 2004. APEC '04. Nineteenth Annual IEEE, vol. 2, pp. 1289-1293 vol.2, 2004.

[4] Y. Ren, M. Xu, K. Yao, Y. Meng, and F. Lee, "Two-stage approach for 12-v vr," Power Electronics, IEEE Transactions on, vol. 19, no. 6, pp. 1498-1506, Nov. 2004

[5] L. Laguna, R. Prieto, O. Garcia, R. Gutierrez, and J. Cobos, "Generalized methodology for the analysis and design of multiphase converters with integrated magnetics," Applied Power Electronics Conference, APEC 2008 - IEEE, 2008

[6] Z. Lu and W. Chen, "Multi-phase inductor coupling scheme with balancing winding in vrm applications," Applied Power Electronics Conference, APEC 2007 - Twenty Second Annual IEEE, pp. 731-735, Feb. 25 2007March 12007.

[7] P. Zumel, O. Garcia, J. A. Cobos, and J. Uceda, "Tight magnetic coupling in multiphase interleaved converters based on simple transformers," Applied Power Electronics Conference and Exposition, 2005. APEC 2005. Twentieth Annual IEEE, vol. 1, pp. 385-391 Vol. 1, 6-10 March 2005.

[8] S. Prabhakaran, C. Sullivan, T. O’Donnell, M. Brunet, and S. Roy, "Microfabricated coupled inductors for dc-dc converters for microprocessor power delivery," Power Electronics Specialists Conference, 2004. PESC 04. 2004 IEEE 35th Annual, vol. 6, pp. 4467-4472 Vol.6, 20-25 June 2004.

[9] M. Xu, Y. Ying, Q. Li, and F. C. Lee, "Novel coupled-inductor multiphase vrs," Applied Power Electronics Conference, APEC 2007 - Twenty Second Annual IEEE, pp. 113-119, Feb. 25 2007-March 12007. 